\title{
Transient Effects in Gain-Clamped Discrete Raman Amplifier Cascades
}

\author{
G. Bolognini and F. Di Pasquale
}

\begin{abstract}
This letter describes the dynamic characteristics of all-optically gain clamped discrete Raman amplifier chains. Amplifier cascades are analyzed under channels add/drop conditions in large bandwidth WDM metro-core applications. Numerical results show that the long Raman fiber length does not allow instantaneous gain clamping giving rise to wide power oscillations in long Raman amplifier chains. A possible way to limit this detrimental effect is suggested and numerically demonstrated.
\end{abstract}

Index Terms-Dynamic response, gain control, optical amplifiers, Raman scattering, transient behavior.

\section{INTRODUCTION}

$\mathbf{T}$ HE USE of Raman fiber amplifiers in WDM systems and networks appears to be very attractive, owing to the wide and flexible amplification bandwidth, the low level of added noise, and the possibility of reducing system penalties due to nonlinear propagation effects. Their use in distributed and discrete configurations allows one to extend the span length, to increase the number of spans, and the overall transmission capacity. In particular, discrete (or lumped) Raman amplifiers (RAs) are attractive for WDM metro-core applications not only for the possibility they offer in terms of large and flexible bandwidth but also because of their interesting saturation characteristics; linear RAs can greatly simplify the network management avoiding complex and expensive gain control techniques. However, when large bandwidth and high number of WDM channels are desirable, the consequent high amplifier output power and pump-to-pump interactions make linear operations difficult to be achieved and unpractical in terms of pump power requirement.

It is then important to study gain control techniques to counteract transient phenomena [1], [2] which can occur due to gain saturation when Raman amplifiers are used in add/drop networks or with burst-mode traffic; temporary variations in the amplifier gain can actually induce system penalties because of optical signal to noise ratio (OSNR) degradations and nonlinear impairments.

In this letter, for the first time to our knowledge, we analyze through numerical simulations the dynamic behavior of gain-clamped multiwavelength pumped discrete Raman amplifier cascades in presence of WDM channels add/drop; all-optical gain control (AOGC) is introduced by gain clamping each RA through an optical feedback which generates lasing oscillation counterpropagating with respect to the WDM signals. Co-

Manuscript received February 19, 2003; revised August 27, 2003.

The authors are with Scuola Superiore Sant'Anna di Studi Universitari e Perfezionamento, 56127 Pisa, Italy (e-mail: gabriele.bolognini@cnit.it).

Digital Object Identifier 10.1109/LPT.2003.818928 propagating configurations, where the laser light travels in the same direction as the signals, are not considered in this work as they can be inconvenient in terms of nonlinear interactions and noise transfer from laser light to signals [3], [4].

We show that the relative long Raman fiber length (order of several kilometers) does not allows instantaneous gain clamping, giving rise to signal power oscillations which can degrade systems performances before reaching steady-state conditions in long amplifier chains. Numerical results point out that over-clamping the Raman amplifiers can reduce these power excursions and effectively improves the gain control mechanism. The proposed gain clamping technique can also be used to adapt the amplifier gain to different span losses without introducing significant spectral gain tilt.

\section{THEORETICAL METHOD}

The time-domain model used for analyzing clamped RA cascades, similar to [1], allows the description of both co-and counterpropagating pumping schemes, takes into account the effects of pump and signal multiple order Raman interactions and signal double Rayleigh scattering (DRS); amplified spontaneous emission (ASE) light has been neglected in order to reduce computational effort. A steady-state model which includes ASE light and their Rayleigh scattering in both co-and counterpropagating directions is also used to investigate the RA noise figure characteristics [5]. Both dynamic and static models allow one to investigate AOGC techniques.

The time-domain partial differential equation for a single amplifier stage, describing the $i$ th signal $\left(i=1 \ldots N_{s}\right)$ or the $i$ th pump $\left(i=1 \ldots N_{P}\right)$ evolution, is given by

$$
\begin{aligned}
\left(\frac{\partial}{\partial z}\right. & \left. \pm \frac{1}{V_{S, P}} \cdot \frac{\partial}{\partial t}\right) P_{S_{i}, P_{i}}^{ \pm}(z, t) \\
= & \mp \alpha_{S_{i}, P_{i}} P_{S_{i}, P_{i}}^{ \pm}(z, t) \pm \gamma_{i} P_{S_{i}, P_{i}}^{\mp}+ \\
& \pm \sum_{j \neq i} C_{i j} P_{S_{i}, P_{i}}^{ \pm} \cdot\left[P_{p_{j}}^{+}+P_{p_{j}}^{-}\right] \\
& \pm \sum_{j \neq i} C_{i j} P_{S_{i}, P_{i}}^{ \pm} \cdot\left[P_{s_{j}}^{+}+P_{s_{j}}^{-}\right]
\end{aligned}
$$

where $P_{S, P}$ indicate the signal/pump power, the superscripts $+/-$ represent the co-and counterpropagating direction, $V_{S, P}$ are the group velocities and $\alpha_{S, P}$ the absorption coefficients for signals/pumps, $\gamma_{i}$ is the Rayleigh backscattering coefficient and $C_{i j}$ indicate the normalized Raman gain coefficient:

$$
C_{i j}=\frac{g_{i j}}{A_{e f f}} \text { for } \lambda_{i}>\lambda_{j}, \quad C_{i j}=-\frac{\lambda_{j}}{\lambda_{i}} \frac{g_{i j}}{A_{e f f}} \text { for } \lambda_{i}<\lambda_{j}
$$




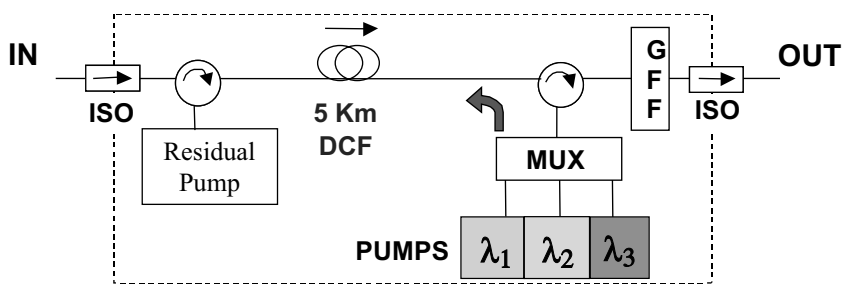

Fig. 1. Scheme of single amplifier stage.

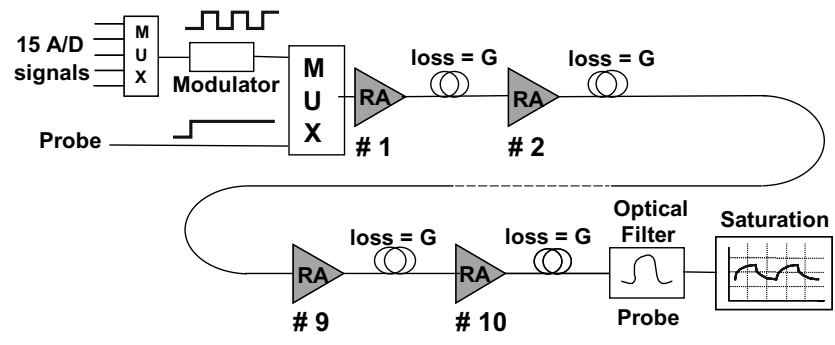

Fig. 2. Cascade of 10 discrete Raman amplifiers.

where $g_{i j}$ is the Raman gain coefficient, and $\mathrm{A}_{\text {eff }}$ is the fiber effective area. The equation describing the counterpropagating lasing light $P_{f}$ is similar to the previous one, apart from the spontaneous emission factor at second member $\left(-2 h \nu_{f} \Delta v \sum C_{f, j}\left(P_{p_{j}}^{+}+P_{p_{j}}^{-}\right)\right)$, and the feedback boundary conditions imposing $P_{f}(t, z=L)=P_{f}\left(t-t_{f}, z=0\right) / F$, where $t_{f}$ and $F$ are respectively the time delay and the attenuation introduced by the feedback line.

\section{RESULTS}

\section{A. Amplifier Cascade Without AOGC}

The scheme of the single amplifier stage used in the simulations is shown in Fig. 1. The amplification bandwidth is about $60 \mathrm{~nm}$ and is obtained by three counterpropagating pump wavelengths centered at 1423, 1445, and $1473 \mathrm{~nm}$. The Raman fiber is dispersion compensating fiber (DCF), whose length $(5 \mathrm{~km})$ has been optimized for the best trade-off between gain, required pump power and DRS impact. For a total input signal power of $0 \mathrm{dBm}$ over the signal bandwidth $1520-1580 \mathrm{~nm}$, the amplifier gain is about $20 \mathrm{~dB}$ and is provided by input pump powers of $0.69,0.28$, and $0.2 \mathrm{~W}$ at 1423,1445 , and $1473 \mathrm{~nm}$, respectively (the gain flatness is $2 \mathrm{~dB}$ ). The absorption coefficients $\alpha_{S}, \alpha_{P}$ have been considered equal to $0.5 \mathrm{~dB} / \mathrm{km}$ and $0.7 \mathrm{~dB} / \mathrm{km}$, respectively. Note that in the amplifier chain simulation a gain flattening filter (GFF) is introduced at the output of each Raman amplifier. The noise figure of each RA is less than $4 \mathrm{~dB}$ throughout the whole bandwidth. Spectral signal-to-DRS noise ratio (S/DRS) at each RA output is higher than $37 \mathrm{~dB}$ and multipath interference noise due to DRS does not induce any penalty at cascade end (S/DRS $>27 \mathrm{~dB}$ at the receiver).

The dynamic properties of the amplifier chain have been investigated under WDM channel add/drop as shown in Fig. 2. In order to analyze the worst case scenario, 15 out of 16 channels $(-12 \mathrm{dBm} / \mathrm{ch}$ input power) were simultaneously added and dropped at the first RA input (at $2.66 \mathrm{kHz}$ rate), while the input

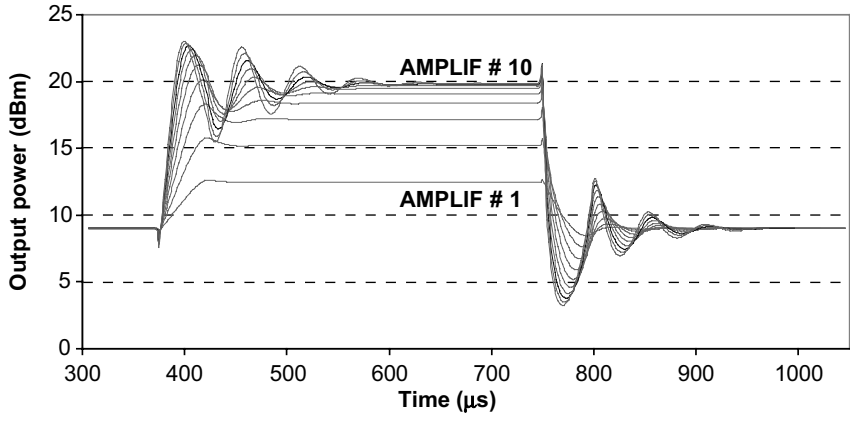

Fig. 3. Output power of probe after each amplifier stage subsequently to the drop and add of $15 / 16$ channels.

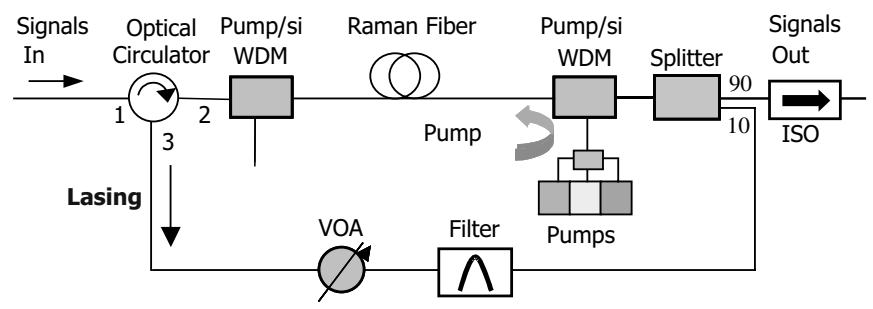

Fig. 4. Counterpropagating AOGC amplifier scheme.

power of the remaining channel (probe), has been kept constant in time. The probe output power variations, due to channels add/drop, are then observed after each amplifier stage. Note that the flattened RA gain with full channel load exactly compensates for the span loss. On the other hand, when 15/16 channels are dropped, the stationary gain of the probe increases, and its output power at each amplifier stage becomes greater and greater along the cascade. Furthermore, before reaching a stationary state, the probe output power experiences transient variations, which accumulate along the chain, and can lead to wide power oscillations.

Fig. 3 shows the probe output power transient behavior after each amplifier stage induced by the drop and following add of $15 / 16$ channels. Note that in order to better compare the transient behavior at the output of each amplifier stage, the temporal scale of each curve in Fig. 3 has been translated in such a way that the instants of channel add/drop coincide for all amplifier stages. The oscillatory transient behavior in Fig. 3 is characterized by two different timescales; a slower one, depending on pump depletion and with a timescale $t_{a m p}$ related to the Raman fiber length ( $\sim 25 \mu$ s for a 5-km-long DCF) and a faster one, depending on intersignal Raman interaction [6]. Note that the power oscillations induced by the drop of 15/16 channels become higher and narrower along the cascade, and after $10 \mathrm{am}$ plifiers can reach a maximum value as high as $13.8 \mathrm{~dB}$.

\section{B. Amplifier Cascade With AOGC}

In this section, we analyze the performance of all-optically gain clamped discrete RA chains in presence of WDM channels add/drop. Fig. 4 shows a schematic structure of the simulated multiwavelength pumped discrete Raman amplifier; gain clamping is achieved by an optical feedback which generates lasing oscillation counterpropagating with respect to the WDM signals. An optical filter (width $0.5 \mathrm{~nm}$ ) selects a fraction of ASE light within the amplifier gain bandwidth, and a variable optical 


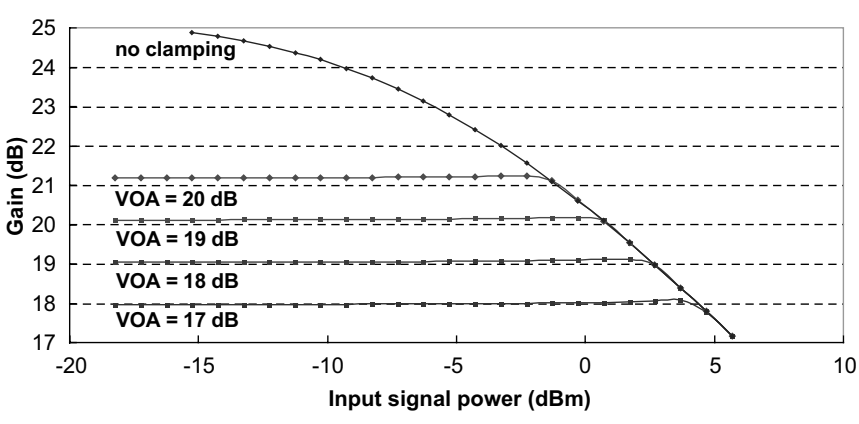

Fig. 5. Single-channel $(1577 \mathrm{~nm})$ amplifier gain versus input signal power, for several values of VOA attenuation.

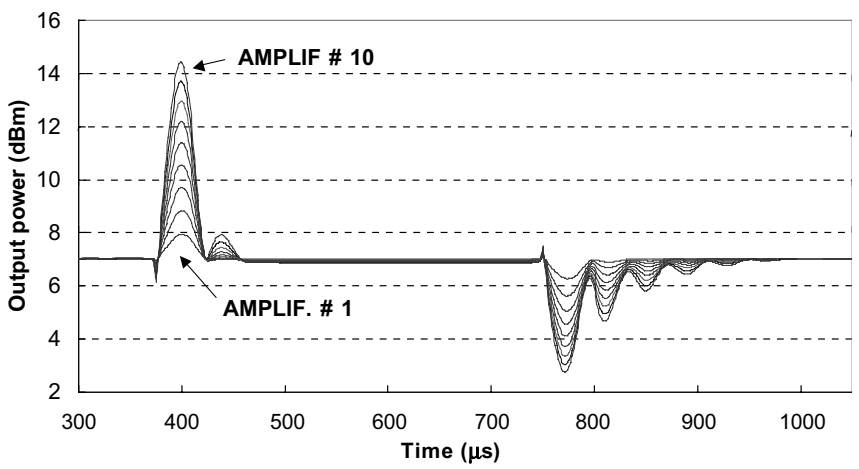

Fig. 6. Oscillations of probe output power along the cascades caused by channel add/drop.

attenuator (VOA) is used to clamp the Raman gain at different values, depending on the particular span loss which should be compensated by the amplifier.

Considering the same pump wavelengths and powers as in the previous section, Fig. 5 shows the static behavior of the gain clamped amplifier with a single channel at $1577 \mathrm{~nm}$ and an optical filter centred at $1582 \mathrm{~nm}$. A static spectral analysis of the amplifier characteristics also showed that the VOA allows one to vary the Raman gain within the signal bandwidth $1520-1580 \mathrm{~nm}$ ( $0 \mathrm{dBm}$ total input signal power) from 17 to $21 \mathrm{~dB}$ without introducing any serious gain tilt $(\sim 1 \mathrm{~dB})$.

Note that, unlike what happens in all-optically gain clamped erbium-doped fiber amplifiers (EDFAs), the small gain tilt introduced by over-clamping discrete Raman amplifiers is mainly due to nonhomogeneous pump depletion and would completely disappear in single wavelength pumped amplifiers.

Fig. 6 shows the probe output power transient behavior after each amplifier stage induced by the drop and following add of 15/16 channels at the input of a ten AOGC amplifier cascade. The input power per channel is $-12 \mathrm{dBm}$ and the VOA is equal to $18 \mathrm{~dB}$ corresponding to an average Raman gain of $19 \mathrm{~dB}$. It is evident that even with AOGC in each amplifier along the cascade, temporary power oscillations take place at each amplifier output before reaching a stationary state; these oscillations are amplified along the cascade and their maximum amplitude becomes $8 \mathrm{~dB}$ high at the last amplifier output. The timescale of these oscillations is mainly related to the fiber transit time $t_{\mathrm{amp}}$ and this suggests that an effective AOGC scheme requires as short as possible Raman fiber lengths.

Moreover, as we have observed that power peaks during oscillations depend on the laser strength within the amplifier im-

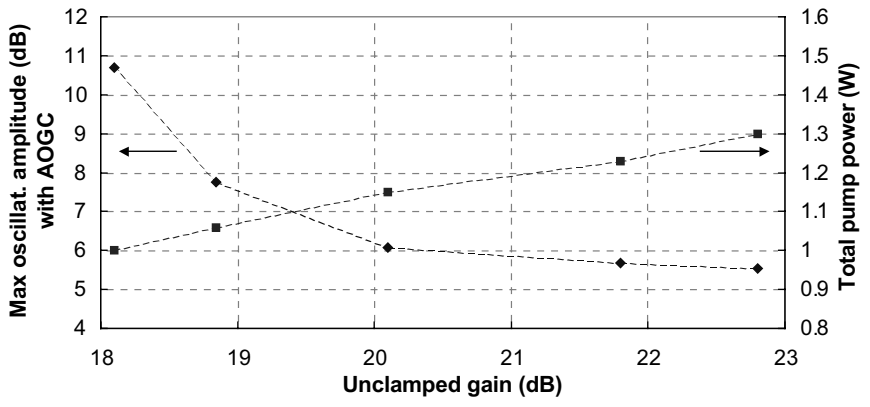

Fig. 7. Maximum amplitude of output probe oscillations at the cascade output (left) and total required pump power (right) versus amplifier unclamped gain in a 18-dB clamped RA.

mediately before the WDM channels add/drop, we have investigated the possibility of reducing these power peaks by overclamping each amplifier along the cascade. Thus we have analyzed the maximum oscillation amplitude along the cascade for different amplifier structures, where the same required Raman clamped gain (with full channels load) is obtained with different VOA values and total pump power. Fig. 7 shows the maximum oscillation amplitude at the amplifier cascade output as a function of the unclamped probe gain with full channels load $(-12 \mathrm{dBm} / \mathrm{ch}$ for a total input power of $0 \mathrm{dBm})$, with the required clamped gain fixed to $18 \mathrm{~dB}$. Fig. 7 also shows the total pump power which is necessary in order to achieve the respective unclamped probe gain. Note that over-pumped amplifiers with higher unclamped gain require more lasing feedback to provide the required gain and consequently operate with the lasing light above threshold even with full channels load. This feature can be exploited to minimize oscillation power peaks along the cascade as clearly shown in Fig. 7. Note that by increasing the unclamped gain from 18 to nearly $22 \mathrm{~dB}$, the maximum oscillation amplitude at the last amplifier output is reduced from about 11 to $6 \mathrm{~dB}$. However this improvement is paid in terms of required pump power (as shown in Fig. 7 as well) which is increased for amplifiers with higher unclamped gain.

In conclusion, we have analyzed transient effects in all-optically gain controlled Raman amplifier cascades under WDM channels add/drop operations. Important issues, such as temporary power swings related to the Raman fiber length and counterpropagating feedback scheme, have been addressed and possible ways to mitigate these detrimental effects have also been suggested.

\section{REFERENCES}

[1] A. Bononi and M. Papararo, "Transient gain dynamics in saturated counter-pumped Raman amplifiers," in OFC2002, Anaheim, CA, Mar. 2002, Paper ThR1, pp. 511-512.

[2] C.-J. Chen and W. S. Wong, "Control of transient effects in distributed and lumped Raman amplifiers," Electron. Lett., vol. 37, no. 21, pp. 1304-1305, 2001.

[3] X. Zhou et al., "Theoretical investigation of fiber Raman amplifier with dynamic gain control," in OFC2001, Anaheim, CA, Mar. 2001, Paper WDD17, pp. WDD17.1-3.

[4] S. S.-H. Yam et al., "Gain-clamped S-band discrete Raman amplifier," in OFC2002, Anaheim, CA, Mar. 2002, Paper ThB4, pp. 385-387.

[5] S. Faralli and F. Di Pasquale, "Impact of double Rayleigh scattering noise in distributed higher order Raman pumping schemes," IEEE Photon. Technol. Lett., vol. 15, pp. 804-806, June 2003.

[6] S. Gray, "Transient gain dynamics in wide bandwidth discrete Raman amplifiers," in OFC2002, Anaheim, CA, Mar. 2002, Paper ThR2, pp. 512-513. 